\title{
Realidad virtual y sus aplicaciones en trastornos mentales: una revisión
}

\section{Virtual reality and its applications in mental disorders: a review}

\author{
Héctor Brito C. ${ }^{1, a}$ y Benjamín Vicente P. ${ }^{2}$
}

\begin{abstract}
The last two decades have witnessed the emergence of virtual reality (VR) as an important tool for research, evaluation and treatment in mental disorders in mental health, creating interactive environments generated by computer, where individuals can repeatedly experience their problematic situations and learn, through psychological treatments based on evidence, how to overcome difficulties. The purpose of this article is to provide an updated systematic review of the literature on the use of VR in mental health problems. A systematic review was made identifying 245 studies, of which only 29 were used related to $R V$ models, of which 18 corresponded to different disorders (anxiety, depression, schizophrenia, psychosis, eating disorders, obsessive compulsive disorder) and 11 to empirical studies. From their analysis it is concluded that the ability of the $R V$ to simulate reality could greatly increase access to therapies in mental disorders while the results could be improved by the ability of technology to create new realities.
\end{abstract}

Key words: Virtual reality (VR); Virtual reality investment; Mental health; Mental disorders. Rev Chil Neuro-Psiquiat 2018; 56 (2): 127-135

\section{Introducción}

$\mathrm{L}$ as tecnologías han ingresado cada vez más al desarrollo de la vida actual ampliando las características evolutivas de los seres humanos, con aplicaciones que ofrecen nuevos escenarios en la resolución de problemas y también de los trastornos mentales. Una de las herramientas que se presentan como una vanguardia tecnológica clínica es la realidad virtual (RV). La RV inversiva crea mundos interactivos generados por computadora, que sustituyen las percepciones sensoriales del mundo real por las generadas digitalmente, produciendo la sensación de estar realmente en nuevos entornos de tamaño real. La RV permite un control estrecho sobre los estímulos presentados y las estrategias de intervención facilitando implementaciones más precisas ${ }^{1}$. De esta forma pueden ser utilizada como una valiosa herramienta en la evaluación, tratamiento e investigación, ya que

Recibido: $18 / 12 / 2017$

Aceptado: 29/05/2018

Los autores no presentan ningún tipo de conflicto de interés.

Doctorado en Salud Mental, Universidad de Concepción, Chile.

2 Departamento de Psiquiatría y Salud Mental, Facultad de Medicina, Universidad de Concepción, Chile.

a Departamento de Kinesiología, Universidad Autónoma de Chile. 
permite la reproducción de ambientes y estímulos controlados $^{2}$, además de poder ser replicados las veces que sea necesario, en diversos contextos.

\section{RV y Problemas de salud mental}

La gran ventaja de la RV es la utilización de la sensación de realidad, en donde la mente y cuerpo se comporta y reacciona como si fuera una situación real, pero sus usuarios saben que es un entorno informático no real; esto permite que sea mucho más fácil enfrentar situaciones complejas a través de la RV, en comparación con las generadas en la vida real, además de probar nuevas estrategias de intervención, con ambientes más controlados, pero permitiendo la verosimilitud de experiencias cotidianas ${ }^{1}$.

Existen múltiples usos potenciales de la RV en los problemas de salud mental, es por esto que se han generado algunas propuestas para identificar modelos y líneas de estudio de diversos trastornos mentales ${ }^{3-5}$.

La utilización de la RV en el diagnóstico estándar de los trastornos mentales, podría transformarse en una herramienta que permitiría mayor validez de los mismos, ya que el diagnóstico es realizado principalmente a través de la recolección de información retrospectiva mediante entrevistas clínicas y cuestionarios validados. Inevitablemente, los seres humanos tienden a ser muy subjetivos en sus puntos de vista. La memoria, por otra parte, es notoriamente falible. Pero la RV permite la observación clínica directa de las conductas en pacientes, en diversos escenarios, los cuales se pueden replicar dependiendo de situaciones específicas y contextos particulares, además de permitir dosificar los estímulos y controlar la frecuencia e intensidad de los mismos ${ }^{3}$.

Una de las variables más utilizadas para medir la eficiencia de la RV, en implementación de diseños de investigación, es el sentido de presencia (SP), la sensación psicológica de "estar allí", que los individuos pueden experimentar en entornos inmersivos de $\mathrm{RV}^{3,6}$. Otro de los modelos utilizados es la encarnación virtual, que permite que un cuerpo virtual de tamaño natural sustituya al cuerpo real de una persona en una realidad virtual inmersiva, con el objetivo que el usuario tenga sensación de propiedad de esta ilusión corporal. Esta ilusión del cuerpo virtual como propio, permite la identificación en primera persona del cuerpo; permite, además, que las modificaciones en las características corporales realizadas en la RV sean incorporadas a la percepción del individuo, configurando la percepción en el cuerpo real ${ }^{7}$. La RV al permitir el diseño controlado del entorno, puede utilizarse para manipular los desencadenantes ambientales, como por ejemplo la angustia que se ha aplicado como estímulo estresor en las personas con problemas de salud mental, lo que permitiría estudiar cómo se desarrolla este proceso y ser utilizado como intervención para manejar de mejor manera sus dificultades ${ }^{8}$.

La RV se ha utilizado en entornos clínicos para tratar una amplia gama de problemas cognitivos, emocionales y motores en diversos trastornos psicológicos y psiquiátricos ${ }^{3}$. La eficacia de la RV se ha verificado en el tratamiento de la acrofobia, fobia a las arañas, trastorno de pánico y agorafobia, alteraciones de la imagen corporal, trastornos de la alimentación compulsiva y el miedo a volar ${ }^{9-12}$. El objetivo de este artículo es proporcionar una revisión sistemática actualizada de la literatura sobre la utilización de la RV en los síntomas y/o trastornos psiquiátricos más prevalentes.

\section{Método}

Se llevó a cabo una revisión sistemática de la utilización de la RV en los trastornos mentales hasta el año 2017, los criterios de inclusión fueron: revisiones, metaanálisis y ensayos clínicos, en donde se aplicara RV, desde la perspectiva de la identificación de las diversas aplicaciones en evaluación, desarrollo de teoría y tratamiento. Los criterios de exclusión fueron el uso de RV, pero sin enfoque específico en un síntoma o trastorno mental; también se excluyeron los trastornos de inicio de la infancia o los efectos de los juegos de $\mathrm{RV}$ que no fueron diseñados como intervenciones y efectos de la RV en población militar (Figura 1). 


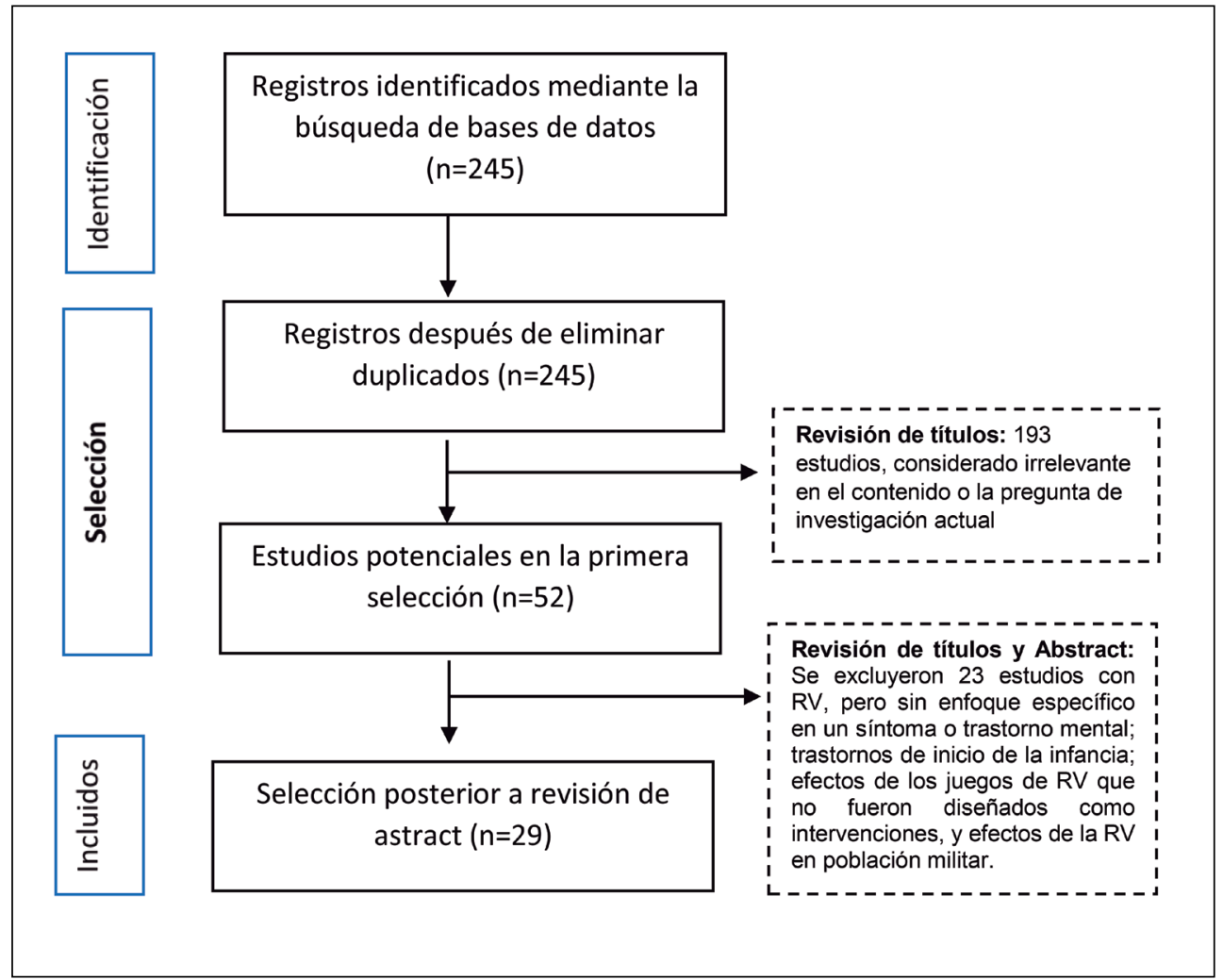

Figura 1. Elementos de informes preferidos para revisiones.

\section{Resultados}

De los 245 estudios, usando los criterios de inclusión y exclusión a través de la revisión de títulos, solo se seleccionaron 52 estudios potencialmente relevantes; posteriormente, luego de la revisión de los abstract se redujo a 29 estudios, los cuales fueron utilizados en esta revisión. Estos fueron clasificados según tipo de síntoma o trastorno mental: tres estudios de ansiedad, tres de depresión, dos de esquizofrenia, tres de psicosis, cinco de trastornos alimenticios, dos de trastorno obsesivo compulsivo y 11 estudios empíricos.

\section{Aplicaciones de la RV en trastornos mentales}

\section{Ansiedad}

Gran parte de los estudios sobre la RV se han referido a la intervención en los trastornos de ansiedad social. En una revisión que buscaba identificar las aplicaciones de RV en trastornos de ansiedad, diagnosticados según entrevista clínica estructurada del DSM-IV, se identificaron $\mathrm{n}=127$ estudios enfocados en tratamiento, $\mathrm{n}=46$ en evaluaciones; y solo 19 dedicados a investigar las causas de la ansiedad. Además, en múltiples estudios, enfocados en otros tipos de trastornos mentales, se ha utilizado la ansiedad como variable estresora o como medida sintomática de resultado ${ }^{13}$.

Uno de los tratamientos habituales para los trastornos de ansiedad es la terapia cognitivoconductual, en la que los pacientes son expuestos a situaciones de ansiedad, generalmente en la vida real o a través de la exposición imaginaria donde se les pide que imaginen una situación a la cual temen. En las dos últimas décadas, el tratamiento de la exposición también se ha ofrecido a través de la realidad virtual ${ }^{14}$, como intervención principal para los trastornos de ansiedad social, en la cual se encuentra un terapeuta presente para guiar a 
la persona. La mayoría de los estudios con RV tendrían una eficacia, comparable con las intervenciones equivalentes, cara a cara ${ }^{13}$.

En la terapia de exposición por RV para los pacientes con trastornos de ansiedad, se crean situaciones de ambiente simulado en tercera dimensión (3D) y situaciones simuladas por ordenador, a través de la estimulación de los sentidos. En el caso de los trastornos de ansiedad social, uno de los diseños más utilizados es el procesamiento emocional, el cual propone esta variable como mecanismo de cambio, de forma de estimular activando una estructura de temor. La exposición en la RV a situaciones que generan esta emoción (miedo) permite incorporar información nueva y correctiva en la estructura de la memoria, llevando a un cambio en la respuesta a esta emoción ${ }^{14}$.

Los diseños utilizados en la RV, el sentido de la presencia (SP), que corresponde a cuán inmerso se ha sentido el paciente en la realidad virtual y a un estímulo en particular, han sido considerados como el principal mecanismo utilizado para validar los diseños de investigación ${ }^{14}$.

En 33 estudios que relacionaron el trastorno de ansiedad y la SP en RV, con un total de 1.196 participantes, con trastornos de ansiedad según DSMIV-TR o versiones anteriores del DSM, se encontró una correlación positiva entre el autorreporte del sentido de la presencia y ansiedad. El análisis de efectos aleatorios mostró un tamaño de efecto medio para la correlación entre sentido de presencia y ansiedad. Los análisis de moderación revelaron que el tamaño del efecto de la correlación difirió entre los diferentes trastornos de ansiedad, con un gran tamaño de efecto en el miedo a los animales y un tamaño de efecto no pequeño para el trastorno de ansiedad social ${ }^{14}$.

Con respecto a la efectividad de los diseños aplicados con RV como una opción de tratamiento viable para los trastornos de ansiedad, se identificó una revisión que evaluó la RV y su efecto en los trastornos de ansiedad, diagnosticados según criterios del DSM-IV, comparando la exposición a tratamientos en contexto real sin RV y con RV. Trece estudios con un total de $\mathrm{n}=397$ sujetos, mostraron un tamaño de efecto medio grande para RV en comparación con el tratamiento sin $\mathrm{RV}$. Además, aparece una tendencia a una relación dosis-respuesta con más sesiones RV mostrando mayores efectos ${ }^{15}$.

\section{Depresión}

En la investigación de RV en depresión se pesquisó la utilización de modelos de autoidentificación, a través de encarnación virtual para aumentar los sentimientos de autocompasión ("self-compassion") en los pacientes con depresión. La autocompasión personal se operacionalizó como autobondad o autoseguridad ${ }^{6}$. El modelo de la encarnación virtual utilizado en este estudio, consiste en un cuerpo virtual de tamaño natural que sustituye al cuerpo real de una persona en una realidad virtual inmersiva, la cual puede ser modificada en apariencia, sexo, edad, color de piel, comunicación gestual etc.; este modelo permite a través de las modificación del cuerpo virtual, asumir percepciones de la ilusión en mi propia percepción real, que en el caso de la depresión, se utiliza para aumentar los sentimientos de compasión de sí mismo ${ }^{16,17}$.

Para la aplicación de estos diseños se utilizaron cuerpos virtuales dentro de la realidad virtual inmersiva, este fue desarrollado en un escenario de 8 minutos en el que 15 pacientes practicaron la entrega de bondad a un cuerpo virtual de un niño llorando, luego experimentaron la recepción de sí mismos en otro cuerpo virtual, en donde ellos eran pacientes encarnados como niños y escucharon las palabras que ellos mismos dijeran al primer cuerpo virtual. Tras tres repeticiones de este escenario los pacientes evidenciaron reducciones significativas en la severidad de la depresión y la autocrítica, así como un aumento significativo en la autocompasión, desde el inicio hasta el seguimiento de 4 semanas y cuatro de ellos mostraron una mejoría clínicamente significativa en la severidad de la depresión (PHQ-9) ${ }^{17}$.

Un modelo de estudio cuasiexperimiental de otro grupo, utilizó un programa de control de estrés basado en RV en personas con trastornos del estado de ánimo, con 22 participantes, diagnosticados con trastorno depresivo mayor $y$ 
trastorno bipolar (con episodio depresivo), de un hospital terciario en Singapur. El programa tenía por objetivo disminuir el nivel de estrés, midiendo el estrés fisiológico a través de la presión arterial, la frecuencia cardíaca, la temperatura de la piel, la intensidad de depresión, la ansiedad y el estrés subjetivo. Se realizaron tres sesiones diarias de una hora que comprendían dos componentes principales: psicoeducación y la práctica de relajación (a través de vídeos de relajación basados en RV). Los participantes que completaron el programa habían reducido significativamente el estrés subjetivo $(t=6,91, p<0,001)$, el nivel de depresión $(\mathrm{t}=5,62, \mathrm{p}<0,001)$, la ansiedad $(\mathrm{t}=5,54$, $\mathrm{p}<0,001)$; la relajación percibida $(\mathrm{F}=26,20$, $\mathrm{p}<0,001)$, la autopercepción del estrés $(\mathrm{F}=13,77$, $\mathrm{p}<0,001)$, además, de identificar un aumento de la temperatura en la piel $(\mathrm{F}=17,71, \mathrm{p}<0,001)^{18}$.

\section{Esquizofrenia}

Con respecto a la utilización de RV en esquizofrenia, se analizó una revisión de 33 artículos de evaluación y tratamiento, identificando tres principales áreas de estudio: cognitivas $(n=15)$, sociales ( $\mathrm{n}=14)$ y perceptuales/sensitivas $(\mathrm{n}=4)$; clasificados a temas en dos campos principales: $\mathrm{n}=27$ de evaluación y $\mathrm{n}=6$ de entrenamiento. Todos los pacientes reclutados en unidades psiquiátricas (ambulatoria y hospitalización). El 100\% de los estudios revisados en este metaanálisis indican que la RV es una herramienta útil para evaluar y tratar a los pacientes con esquizofrenia evaluados a través de los signos positivos y negativos. Las evaluaciones de las habilidades cognitivas, sociales, perceptuales y sensoriales realizadas por la RV muestran correlaciones positivas con los resultados obtenidos de las evaluaciones con herramientas clásicas (por ejemplo, la Prueba de clasificación de tarjetas de Wisconsin WCST y la escala de comportamiento social SBS). En los seis estudios que utilizaron RV para el entrenamiento cognitivo y habilidades sociales, hubo una mejora en los resultados del rendimiento, en comparación con lo obtenido con programas clásicos sin RV (por ejemplo, la batería para la evaluación del estado neuropsicológico RBANS) ${ }^{19}$.
En un estudio piloto de 12 pacientes, que cumplían con los criterios del DSM-IV-TR para esquizofrenia o trastorno esquizoafectivo, se utilizó un programa integrado que realizó capacitación de las habilidades sociales en RV. El programa de RV permite a los usuarios practicar interacciones sociales con avatares virtuales, los cuales consisten en personas creadas por ordenador incorporados a la realidad virtual para la interacción social; estos tienen por objetivo fomentar el aprendizaje progresivo del repertorio de habilidades sociales y proporcionar refuerzo positivo o negativo. Los resultados revelaron una mejora significativa en los síntomas negativos, la psicopatología, la ansiedad social y la incomodidad, la evitación y el funcionamiento social. Las puntuaciones objetivas obtenidas mediante el uso del programa de RV mostraron un patrón de aprendizaje en la percepción emocional, comportamientos asertivos y tiempo de conversación, las que se mantuvieron al seguimiento de cuatro meses ${ }^{20}$.

\section{Psicosis}

Algunos estudios han utilizado la RV para evaluar las experiencias psicóticas, como los procesos psicológicos y mecanismos asociados a la psicosis, con el fin de comprender las variables asociadas, investigando los efectos de la sensibilidad interpersonal, la victimización infantil de intimidación, el asalto físico, la discriminación étnica percibida, la derrota social, la densidad de población y la densidad étnica, en modelos de evaluación en tiempo real. Otros estudios demostraron el potencial de la RV para investigar la ideación paranoide, las experiencias anómalas, la autoconfianza, la autocomparación, la activación fisiológica y la respuesta conductual $^{21}$.

En un estudio experimental se buscó evaluar en RV el efecto de la sensibilidad al estrés social ambiental en la psicosis. Los estresores sociales virtuales eran la densidad de la población, la densidad étnica y la hostilidad. La paranoia y la angustia subjetiva aumentaron con el grado de estrés social en el medio ambiente. La esquizotipia de la psicosis y los síntomas preexistentes, en particular el afecto negativo, impactaron positivamente el nivel de pa- 
ranoia y angustia en respuesta al estrés social. Estos resultados proporcionan evidencia experimental de que la mayor sensibilidad al estrés social ambiental puede jugar un papel importante en la aparición y evolución de la psicosis ${ }^{22}$.

Otro estudio experimental incorporó 52 pacientes con trastornos psicóticos, 40 hermanos de pacientes con trastorno psicótico y 47 controles. Los sujetos fueron expuestos a cafés virtuales, con 40 avatar hostiles y 86 neutrales, en donde también se evaluó el nivel de estrés social, en términos de hacinamiento, etnicidad y hostilidad. Se demostró que la distancia interpersonal aumentó cuando los estresores sociales estaban presentes en el ambiente. No se encontró ninguna diferencia en la regulación interpersonal de la distancia entre los grupos. La ansiedad social y la angustia se asociaron positivamente con la distancia interpersonal en la muestra total ${ }^{23}$.

\section{Trastornos alimentarios}

Se realizó un estudio controlado aleatorizado con seguimiento de un año, en 90 pacientes obesas para probar a corto y largo plazo la eficacia clínica de una terapia cognitiva-conductual mejorada, incluyendo la RV como protocolo dirigido a desbloquear la memoria negativa del cuerpo de los pacientes obesos mórbidos con trastornos de atracones, posterior a una hospitalización.

Los ambientes de RV presentaron situaciones críticas relacionadas con los mecanismos de mantenimiento/recaída (hogar, supermercado, pub, restaurante, piscina, playa, gimnasio) y dos áreas de comparación de imágenes corporales. A través de la experiencia en $\mathrm{RV}$, los pacientes practicaron gestión y toma de decisiones generales y habilidades para resolver problemas. Mediante la práctica directa de estas habilidades en el entorno de RV, los pacientes fueron ayudados en el desarrollo de estrategias específicas para evitar y/o hacer frente a situaciones desencadenantes. Los resultados mostraron pérdida de peso al año de seguimiento. Por el contrario, los participantes en el grupo, no intervenido, recuperaron en promedio la mayor parte del peso que habían perdido durante el programa de hospitalización ${ }^{24}$.
En el caso de la anorexia nerviosa, se ha utilizado la RV bajo el supuesto de la experiencia distorsionada persistente del tamaño de su cuerpo, en donde se ha investigado si una ilusión del cuerpo completo afecta la estimación del tamaño corporal de las partes del cuerpo, con estimulación visotáctil sincrónica. Los pacientes disminuyeron su estimación sobre el tamaño corporal en hombros abdomen y cadera, generando un efecto más fuerte en la estimación de la circunferencia abdominal ${ }^{25}$. Estudios con similares resultados orientan el potencial de este diseño, para nuevas investigaciones que nos permitan mayor comprensión de la ilusión de la propiedad del cuerpo en la RV, ya que esto podría mejorar los tratamientos de los trastornos alimentarios $^{26,27}$.

En otro estudio se evaluó la efectividad de la RV como una estrategia de segundo nivel de tratamiento para 64 pacientes con bulimia nerviosa (BN) y trastorno por atracón (TA) que habían sido tratados y que cumplieron con los criterios diagnósticos del DSM-5. El diseño de investigación realizado en este estudio permite la creación jerárquica de un software a exposición virtual individualizada combinando los cuatro entornos virtuales y los diez alimentos que produjeron los niveles más altos de antojo de alimentos para un total de 40 situaciones interactivas 3D (medio ambiente y alimentos). Luego, los participantes, fueron expuestos a estos 40 ambientes e indicaron el nivel de ansiedad, presentando diferencias significativas observadas entre los dos grupos, $\mathrm{BN} \mathrm{y}$ TA, en el postratamiento en aspectos dimensionales (comportamiento y actitud, ansiedad, antojo alimentario) y categóricos (tasas de abstinencia), con $\mathrm{RV}^{28}$.

\section{Trastorno obsesivo-compulsivo}

La RV se ha utilizado como un complemento a la terapia conductual del TOC, utilizando como variable de resultado, las medidas basales de síntomas obsesivos y compulsivos. Los resultados indicaron que los participantes con TOC mostraron una disminución significativa tanto en los niveles globales de depresión como en las obsesiones y compulsiones. 
Dentro de los modelos aplicados también se ha introducido la utilización de escenarios que generen ansiedad en los pacientes con TOC, comparado con controles sanos. El grado de ansiedad en los individuos con TOC se correlacionó positivamente con sus puntuaciones de síntomas y puntuaciones de tendencia inmersiva. Lo que sugiere la posibilidad de que la RV tenga un valor como modelo para la estimulación de ansiedad o herramienta de tratamiento para el TOC $^{29}$.

En un estudio experimental donde se usó la RV en el tratamiento basado en la inferencia de la acumulación compulsiva, en pacientes con diagnóstico primario de TOC por acaparamiento compulsivo, según DSM-IV-TR, se realizaron 3 sesiones en RV; en un ambiente simulado, confeccionado como una réplica de las habitaciones de los participantes, la cual se obtuvo a través de fotografías en 360 grados, lo que permitió construir una realidad virtual inmersiva. Los sujetos debían seleccionar y eliminar objetos de sus hogares, simulada por RV, en una lata de basura virtual en $3 \mathrm{D}$, registrando el nivel de ansiedad generado. Se encontraron cambios significativos en las medidas de ansiedad comparados con la medición base y con el grupo control $^{30}$.

\section{Conclusiones finales}

La capacidad de la RV para simular la realidad podría aumentar en gran medida el acceso a las terapias en los trastornos mentales, ya que la tecnología digital se ha convertido en una parte integral de nuestra vida diaria. La adaptación de la terapia a estas formas de implementación permite un tratamiento más personalizado (tratamiento correcto, para la persona adecuada en el momento adecuado); y altamente participativo (entornos interactivos y receptivos). Aumentar el uso de la RV en la práctica clínica ayudará a abordar algunas de las limitaciones metodológicas de los estudios actuales, además de incorporar nuevos modelos de estudio a través de la ilusión, la encarnación virtual, la sensación de presencia o realidad percibida y la inmersión cada vez más sofisticada.

\section{Resumen}

Las dos últimas décadas han sido testigos de la aparición de la realidad virtual (RV) como una herramienta importante para la investigación, evaluación y tratamiento en los trastornos mentales en la salud mental, creando entornos interactivos generados por computadora, donde los individuos pueden experimentar repetidamente sus situaciones problemáticas y aprender, a través de tratamientos psicológicos basados en la evidencia, cómo superar dificultades. El propósito de este artículo es proporcionar una revisión sistemática actualizada de la literatura sobre la utilización de la $R V$ en los problemas de salud mental. Se realizó una revisión sistemática identificando 245 estudios, de los cuales se utilizaron solo 29 relacionados con modelos de $R V$, de los cuales 18 correspondían a distintos trastornos (ansiedad, depresión, esquizofrenia, psicosis, trastornos alimenticios, trastorno obsesivo compulsivo) y 11 a estudios empíricos; de su análisis se concluye que la capacidad de la RV para simular la realidad podría aumentar en gran medida el acceso a las terapias en los trastornos mentales mientras que los resultados podrían ser mejorados por la capacidad de la tecnología para crear nuevas realidades.

Palabras clave: Realidad virtual(RV); Realidad virtual inversiva; Salud mental; Trastornos Mentales. 


\section{Referencias bibliográficas}

1. Mohr DC, Burns MN, Schueller SM, Clarke G, Klinkman M. Behavioral Intervention Technologies: Evidence review and recommendations for future research in mental health. Gen Hosp Psychiatry 2013; 35 (4): 332-8. Available from: http://dx.doi.org/10.1016/j.genhosppsych.2013.03.008.

2. Maples-Keller JL, Bunnell BE, Kim S-J, Rothbaum BO. The Use of Virtual Reality Technology in the Treatment of Anxiety and Other Psychiatric Disorders. Harv Rev Psychiatry 2017; 25 (3): 103-13. Available from: http://insights.ovid.com/crossref? an $=00023727-201705000-00003$.

3. Valmaggia LR, Latif L, Kempton MJ, Rus-Calafell M. Virtual reality in the psychological treatment for mental health problems: An systematic review of recent evidence. Psychiatry Res 2016; 236: 18995. Available from: http://dx.doi.org/10.1016/j. psychres.2016.01.015.

4. Riva G, Wiederhold BK. Introduction to the special issue on virtual reality environments in behavioral sciences. IEEE Trans Inf Technol Biomed 2002; 6 (3): 193-7.

5. Freeman D. Studying and treating schizophrenia using virtual reality: A new paradigm. Schizophr Bull 2008; 34 (4): 605-10.

6. Bouchard S, Fournier T, Renaud P, Slater M. Letter to the Editor Presence and Emotions 2004; 7 (1): 2004.

7. Petkova VI, Khoshnevis M, Ehrsson HH. The perspective matters! Multisensory integration in egocentric reference frames determines full-body ownership. Front Psychol 2011; 2 (3): 1-7.

8. Kim GJ. A SWOT Analysis of the Field of Virtual Reality Rehabilitation and Therapy. Presence 2005; 14 (2): 119-46.

9. Riva G. Applications of virtual environments in medicine. Methods Inf Med [Internet] 2003; 42 (5): 524-34.

10. Norcross JC, Pfund RA, Prochaska JO. Psychotherapy in 2022: A Delphi poll on its future. Prof Psychol Res Pract 2013; 44 (5): 363-70. Available from: http://doi.apa.org/getdoi.cfm?doi=10.1037/ a0034633.
11. Srivastava K, Chaudhury S, Das R. Virtual reality applications in mental health: Challenges and perspectives. Ind Psychiatry J 2014; 23 (2): 83. Available from: http://www.industrialpsychiatry. org/text.asp?2014/23/2/83/151666.

12. Mishkind MC, Norr AM, Katz AC, Reger GM. Review of Virtual Reality Treatment in Psychiatry: Evidence versus Current Diffusion and Use. Curr Psychiatry Rep 2017; 19 (11): 80. Available from: http://link.springer.com/10.1007/s11920-0170836-0.

13. Freeman D, Reeve S, Robinson A, Ehlers A, Clark D, Spanlang B, et al. Virtual reality in the assessment, understanding, and treatment of mental health disorders. Psychol Med 2017; 1-8. Available from: https://www.cambridge.org/core/product/identifier/S003329171700040X/type/journal_article.

14. Ling Y, Nefs HT, Morina N, Heynderickx I, Brinkman W-P. A Meta-Analysis on the Relationship between Self-Reported Presence and Anxiety in Virtual Reality Exposure Therapy for Anxiety Disorders. Slater M, editor. PLoS One 2014; 9 (5): e96144. Available from: http://dx.plos. org/10.1371/journal.pone.0096144.

15. Powers MB, Emmelkamp PMG. Virtual reality exposure therapy for anxiety disorders: A metaanalysis. Vol. 22, Journal of Anxiety Disorders. Pergamon; 2008; 22 (3): 561-9. Available from: http://www.sciencedirect.com/science/article/pii/ S088761850700103X.

16. Blatt SJ, Zuroff DC. Interpersonal relatedness and self-definition: Two prototypes for depression. Clin Psychol Rev 1992; 12 (5): 527-62.

17. Falconer CJ, Rovira A, King JA, Gilbert P, Antley A, Fearon $\mathrm{P}$, et al. Embodying self-compassion within virtual reality and its effects on patients with depression. Br J Psychiatry Open 2016; 2 (1): 74-80. Available from: http://bjpo.rcpsych.org/lookup/ doi/10.1192/bjpo.bp.115.002147.

18. Shah LBI, Torres S, Kannusamy P, Chng CML, He HG, Klainin-Yobas P. Efficacy of the virtual realitybased stress management program on stress-related variables in people with mood disorders: The feasibility study. Arch Psychiatr Nurs 2015; 29 (1): 6-13. Available from: http://dx.doi.org/10.1016/j. apnu.2014.09.003. 
19. Macedo M, Marques A, Queirós C. Virtual reality in assessment and treatment of schizophrenia: a systematic review. J Bras Psiquiatr 2015; 64 (1): 70-81. Available from: http://www.scielo. $\mathrm{br} /$ scielo.php?script=sci_arttext\&pid=S004720852015000100070\&lng=en\&tlng=en.

20. Rus-Calafell M, Gutiérrez-Maldonado J, RibasSabaté J. A virtual reality-integrated program for improving social skills in patients with schizophrenia: A pilot study. J Behav Ther Exp Psychiatry. 2014; 45 (1): 81-9. Available from: http://dx.doi. org/10.1016/j.jbtep.2013.09.002.

21. Valmaggia LR, Day F, Rus-Calafell M. Using virtual reality to investigate psychological processes and mechanisms associated with the onset and maintenance of psychosis: a systematic review. Soc Psychiatry Psychiatr Epidemiol 2016; 51 (7): 921-36.

22. Veling W, Pot-Kolder R, Counotte J, Van Os J, Van Der Gaag M. Environmental Social Stress, Paranoia and Psychosis Liability: A Virtual Reality Study. Schizophr Bull 2016; 42 (6): 1363-71.

23. Geraets CNW, van Beilen M, Pot-Kolder R, Counotte J, van der Gaag M, Veling W. Social environments and interpersonal distance regulation in psychosis: A virtual reality study. Schizophr Res 2016; 6-11. Available from: http://dx.doi. org/10.1016/j.schres.2017.04.034.

24. Cesa GL, Manzoni GM, Bacchetta M, Castelnuovo G, Conti S, Gaggioli A, et al. Virtual reality for enhancing the cognitive behavioral treatment of obesity with binge eating disorder: Randomized controlled study with one-year follow-up. J Med
Internet Res 2013; 15 (6): e113. Available from: http://www.ncbi.nlm.nih.gov/pubmed/23759286.

25. Keizer A, Van Elburg A, Helms R, Dijkerman HC. A virtual reality full body illusion improves body image disturbance in anorexia nervosa. PLoS One 2016; 11 (10): 1-21.

26. Normand JM, Giannopoulos E, Spanlang B, Slater M. Multisensory stimulation can induce an illusion of larger belly size in immersive virtual reality. PLoS One 2011; 6 (1).

27. Maselli A, Slater M. The building blocks of the full body ownership illusion. Front Hum Neurosci. 2013; 7 (3): 1-15. Available from: http://journal. frontiersin.org/article/10.3389/fnhum.2013.00083/ abstract.

28. Ferrer-García M, Gutiérrez-Maldonado J, PlaSanjuanelo J, Vilalta-Abella F, Riva G, Clerici M, et al. A Randomised Controlled Comparison of Second-Level Treatment Approaches for TreatmentResistant Adults with Bulimia Nervosa and Binge Eating Disorder: Assessing the Benefits of Virtual Reality Cue Exposure Therapy. Eur Eat Disord Rev 2017; 25: 479-90. Available from: http://doi.wiley. com/10.1002/erv.2538.

29. Kim K, Kim CH, Kim SY, Roh D, Kim SI. Virtual reality for obsessive-compulsive disorder: Past and the future. Psychiatry Investig 2009; 6 (3): 115-21.

30. St-Pierre-Delorme M-E, O'Connor K. Using Virtual Reality in the Inference-Based Treatment of Compulsive Hoarding. Front Public Heal 2016; 4 (7): 149. Available from: http://www.ncbi.nlm.nih. gov/pubmed/27486574.

Correspondencia:

Benjamín Vicente MD, PhD,

Departamento de Psiquiatría y Salud Mental,

Facultad de Medicina, Universidad

de Concepción,

Casilla 160-C, Concepción-Chile.

Fono-fax: 56-41-321799/204299

E-mail: bvicent@udec.cl 